\title{
Wheel-rail wear index prediction considering multiple contact patches
}

\author{
J. Santamaria", E.G. Vadillo, O. Oyarzabal \\ Department of Mechanical Engineering. University of the Basque Country. Alda. Urquiso s/n 48013 Bilbao, Spain
}

NOTICE: this is the author's version of a work that was accepted for publication in Wear. Changes resulting from the publishing process, such as peer review, editing, corrections, structural formatting, and other quality control mechanisms may not be reflected in this document. Changes may have been made to this work since it was submitted for publication.

A definitive version was subsequently published in WEAR, VOL. 267, ISSUES 5-8, pp. 1100-1104, 2009 doi:10.1016/j.wear.2008.12.040

The final version can be obtained online from:

http://www.sciencedirect.com/science/article/pii/S0043164809000593

Please cite this paper as: Santamaria, J., Vadillo, E.G., Oyarzabal, O. Wheel-rail wear index prediction considering multiple contact patches. Wear, Vol. 267, pp. 1100-1104. 2009

*Corresponding author. Tel.: +34 94601 4230; fax: +34 946014215.

E-mail address: javier.santamaria@ehu.es (Javier Santamaria) 


\title{
Wheel-rail wear index prediction considering multiple contact patches
}

\author{
J. Santamaria*, E.G. Vadillo, O. Oyarzabal \\ Department of Mechanical Engineering. University of the Basque Country. Alda. Urquiso s/n 48013 Bilbao, Spain
}

\begin{abstract}
This paper shows a new wheel-rail contact model specially suited to obtain wear indexes between wheel and rail when the vehicle negotiates tight curves. The model presented in the paper enables introduction of the two contact point situation using three-dimensional analysis of surfaces including the influence of the angle of attack. The proposed model is based on the generation of look-up tables that are used during the dynamic simulation of the vehicle.
\end{abstract}

Keywords: Wheel/Rail contact, Rail Wear

\section{Introduction}

In railway dynamics [1], solving the problem of wheel-rail contact constitutes a key step in obtaining results in relation to the vehicle's dynamic behaviour, ride safety and wear levels, among other factors. Precise calculation of wheel-rail contact parameters requires a high computational cost. Since this calculation has to be made in each step of the time integration, simplifying hypotheses must be adopted to allow the problem to be dealt with from a practical perspective. One of the most common simplifications is to assume that the point of contact is located over the wheel's vertical diametral section, thereby solving the geometrical problem in two dimensions. This means disregarding the influence of the angle of attack (the angle rotated by the wheelset around the vertical axis with respect to the centerline of the track). Nevertheless, the angle of attack parameter can occasionally be high (e.g. on very sharp underground or tram line curves), and in such cases it could influence localisation of the contact point, which moves away from the vertical plane containing the centerline of the wheel (Figure 1a). This displaced position of the contact point considerably affects computation of the wear indices on materials or estimations of ride safety. On other occasions the contact problem is solved three-dimensionally, assuming a

*Corresponding author. Tel.: +34 94601 4230; fax: +34 946014215 .

E-mail address: javier.santamaria@ehu.es (Javier Santamaria) 


\section{Javier Santamaria / Wear}

certain normal force between the solids, which allows the results to be tabulated in simple format prior to analysis. However, the normal force, in each wheel-rail contact is subject to substantial variations during dynamic simulation of the vehicle, and therefore these tabulated data lose a certain amount of accuracy in such cases.

Another situation that arises frequently in wheel-rail contact and makes solving the problem much more complex is the double contact point. At certain positions of the wheel on the rail two contact patches are produced simultaneously. Creepages and normal forces appear separately in each contact area. A typical double contact point scenario when the rail vehicle is negotiating a tight curve is shown in Figure 1b: when the wheelset is rotated at a large yaw angle and laterally displaced, one contact patch is created on the wheel thread and another on the wheel flange, and the flange contact is displaced from the vertical diametral section. The consideration that two separate contact patches exist, and also that the flange contact patch is longitudinally displaced, is crucial for an accurate estimate of the wear indices that will be produced in these situations. It is obvious that the forces and creepages obtained by considering the forward longitudinal wheel flange contact point differ considerably from those that would be obtained by solving the problem in 2-D or assuming that only one equivalent contact patch is produced.
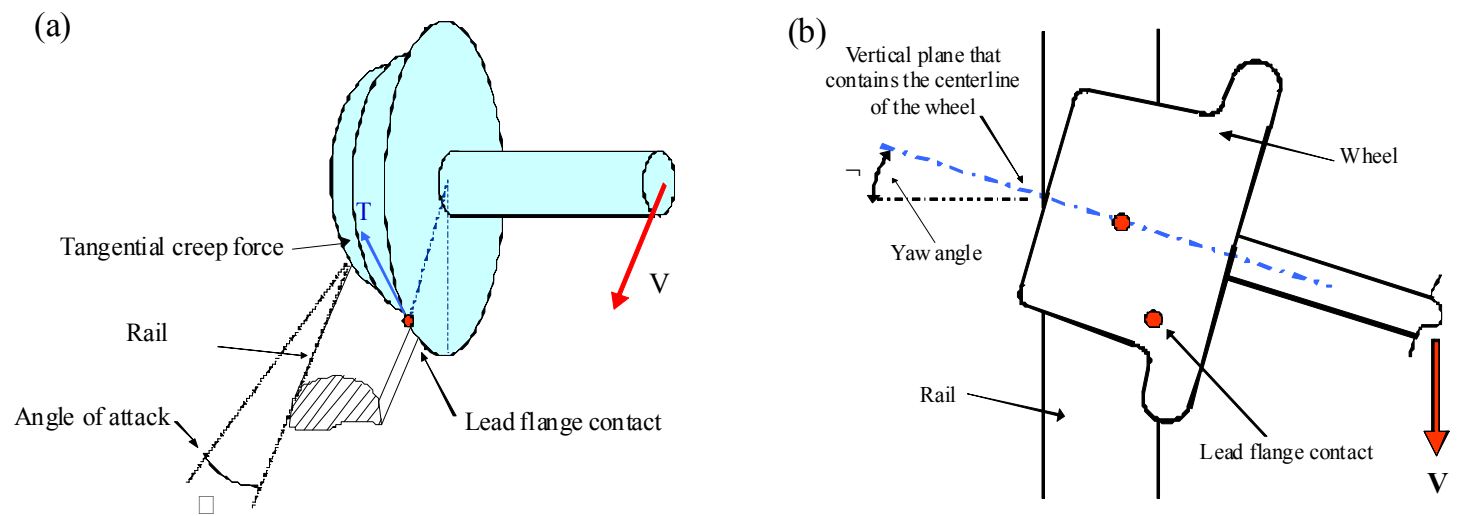

Fig. 1. (a) Wheel-rail contact point when the wheelset rotates at yaw angle $\alpha$; (b) top view of the double contact point scenario with a large yaw angle. The contact patch on the wheel thread is located on the wheel's vertical diametral section, whereas the flange contact patch is displaced longitudinally.

This paper presents a method to solve the wheel-rail contact problem that deals with the double contact point in 3-D with a view to dynamic simulation of the railway vehicle and wear calculation. The method is based on contact look-up tables to store the contact parameters required for simulation with a sufficiently high number of relative positions between the wheel profile and the rail profile. This method does not make any simplifications in relation to settings for wheel profile areas where contact patches may arise or in relation to considerations of a given normal force to calculate contact patches. These characteristics make it an especially useful method for 
determining wear indices when rail vehicles negotiate tight curves, frequently producing double contact point scenarios with large yaw angles. In these situations wear of materials is particularly important [2]. At the end of the paper the results will be provided for a real case study of a vehicle travelling over a tight curve, with the wear index calculated in both 2-D and 3-D with a worn rail profile measured experimentally.

\section{Description of the method}

Of the 6 degrees of freedom (DOF) establishing the position of the wheelset in space as a rigid solid, only 4 condition the relative position between the wheel and the rail, and therefore they condition the contact problem. These 4 DOF are lateral displacement ' $y$ ', yaw angle ' $\alpha$ ', vertical displacement ' $z$ ' and roll angle ' $\phi$ ', as shown in Figure 2a. If the rigid contact hypothesis is considered (the elasticity of the solids is disregarded), this introduces a kinematic restriction on wheelset movement, making two of these DOF independent of the other two. The first two are generally considered as independent [3]. Rigid contact, however, does not allow multi-contact scenarios with two separate contact patches to be examined, and these are of particular importance when a vehicle is moving over curve track.
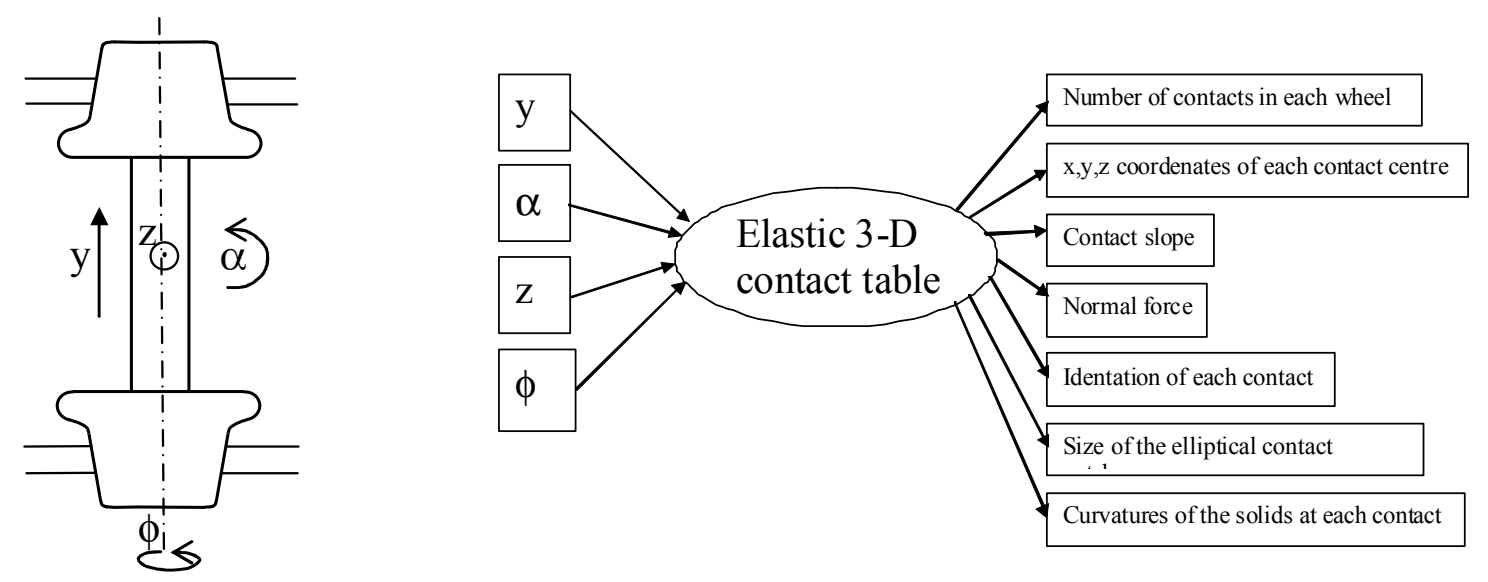

Fig. 2. (a) Degrees of freedom; (b) inputs and outputs of the look-up table with the elastic 3-D contact data.

Treatment of the multi-contact scenarios means taking account of the actual elasticity of the materials. When two elastic solids are forced against each other, their respective centers of gravity move closer as a result of the elastic deformation of areas in proximity to contact. If the wheel and rail profiles are superimposed as rigid solids, interpenetration areas are obtained which are not real, but constitute the basis for solving the problem of contact, both in terms of determining the number and position of the contact patches thus emerging, and the normal force 


\section{Javier Santamaria / Wear}

transmitted in each. The distribution of forces between the contact points depends on elasticity in the contact areas.

The elastic contact calculation between wheel and rail is far more expensive in computational terms than rigid contact, and even more so with three-dimensional analysis. This three-dimensional analysis usually slows down the vehicle's dynamic simulation considerably. However, a look-up table with contact solutions for specific wheelset positions relative to the track can be produced, as in the method proposed in this paper.

If certain values are established for the four independent DOF ' $y$ ',' $\alpha$, ', $z$ ' and ' $\phi$ ', generally speaking a singlepoint contact will not occur, but rather there will be one or several interpenetration areas between each wheel and rail. The method developed seeks the interpenetration areas produced for a specific combination of wheelset DOF and, based on the Hertz theory [4], calculates the normal force between the two bodies that would produce this interpenetration on each of the contact patches. The phenomenon of double contact point between wheel and rail can be resolved in a simple manner, identifying the two interpenetration areas and calculating the normal forces separately.

To solve the elastic contact problem, the wheel profile is placed over the rail profile in accordance with the values assigned to the four DOFs mentioned above. To model the profiles, discrete definer points are used, with an approximate separation of between 0.5 and $3 \mathrm{~mm}$, and also interpolating spline equations. A sweep is carried out following the wheel surface in 3-D, thereby delimiting the areas where interpenetration has taken place. In each of these areas, the cubic spline curves are used to calculate the maximum penetration in normal direction to the wheel profile. According to Hertz's theory, using the value of maximum penetration, the solids' curvature radii at this point, and the material's elastic constants, it is possible to obtain the normal force that must be applied to produce this approach between the two bodies. The ratio between force and penetration is non-linear, and is encountered in the well-known expression $\mathrm{N}=\mathrm{C} \cdot \delta^{3 / 2}$, where $\mathrm{N}$ is the normal force, $\delta$ is penetration, and $\mathrm{C}$ is a constant depending on the elastic properties and curvatures of the solids.

During the dynamic simulation phase, when the normal forces have been obtained in each contact found, the other forces are obtained and the accelerations calculated for all the DOF on the model. This includes the wheelset DOF ' $z$ ' and ' $\phi$ ', which are now independent of the rest and integrated in time like the rest. A numerical integration method is used to obtain the values of the DOF at a subsequent instant, and so the process of superimposing profiles, searching for contacts and obtaining normal forces may be repeated.

The method presented in this paper seeks to precalculate a table with the results obtained for a set of values for the $4 \mathrm{DOF}$ affecting the 3-D problem. This is therefore a tetradimensional table, whose inputs are ' $y$ ', ' $\alpha,{ }^{\prime}$ ' and ' $\phi$ ', and whose outputs are the parameters of the contacts produced for these DOF values (Figure 2b).

In order to include all possible positions that the wheelset could reach during a dynamic simulation, it is necessary to calculate the wheel-rail contact data for a large number of cases, where each case is related to a 


\section{Javier Santamaria / Wear}

certain location of the wheel over the rail. During the dynamic simulation, the elastic contact information at each step of integration is obtained by interpolating the adjacent data in the table. The number of discrete positions calculated in the tables must be sufficient to detect the two-point contact cases if these can occur. For example, when the wheelset moves laterally and the contact point is located at the flange of the wheel instead of its thread, two-point contact will probably occur for certain lateral displacements and angles of attack (' $y$,' $\alpha$ ') of the wheelset. The size of the table should be sufficiently ample to include realistic cases of two-point contact for these displacements. In this way, their interpolation will be possible during simulation.

In order to reduce the size of these tables and their calculation time, a pre-analysis is performed of the profiles, first solving the problem of rigid contact. Since the value of penetrations on contact is always very low compared to the displacements of the wheelset as it moves laterally over the track, the position of the wheelset in this situation is taken as a central reference to vary DOF ' $z$ ' and ' $\phi$ ' in a bid to find the various contact situations [5].

\section{Estimate of wheel-rail wear}

The wear that inevitably occurs between the wheel and the rail while the vehicle is moving depends on a large number of factors, among which sliding phenomena inside the contact patch, the normal force transmitted, the friction coefficient (lubrication conditions), size and shape of the contact patch etc. Wear on wheels and rails makes it necessary for equipment to be replaced when the upper safety limits have been breached and, as a general rule, the vehicle also sustains losses in terms of dynamic performance. Worn profiles tend to be less stable and show lower performance levels when negotiating curved tracks, and this makes reducing the wear index a major factor in the design of railway vehicles. At present there are a number of models attempting to quantify the wear index that include some of the abovementioned parameters. In any case, first it is essential to solve the contact problem. This leads to correct location of all contact patches and provides knowledge as to the size and shape of each patch, data which are essential for the application of any wear model.

Most used theories in railway dynamics assume that wear is proportional to the energy dissipated within the contact patch, calculated as the scalar product of tangential force on the contact and the value of creepage [1, 6-7]. Some experiments show that there is a good correlation between the amount of wear measured in wheels and rails and this frictional energy divided by the contact area [8-9]. The proportionality constant varies depending on wear severity, and the wear rate can be obtained as [8]: 


$$
\begin{array}{ll}
T \nu / A<10.4 \mathrm{~N} / \mathrm{mm}^{2} & \Rightarrow W=5.3 T \nu / A \mu g / \mathrm{m} / \mathrm{mm}^{2} \\
10.4 \leq T \nu / A<77.2 \mathrm{~N} / \mathrm{mm}^{2} & \Rightarrow W=55.0 \mu \mathrm{g} / \mathrm{m} / \mathrm{mm}^{2} \\
T \nu / A \geq 77.2 \mathrm{~N} / \mathrm{mm}^{2} & \Rightarrow W=55.0+61.9(T \nu / A-77.2) \mu \mathrm{g} / \mathrm{m} / \mathrm{mm}^{2}
\end{array}
$$

where $T$ is creep force at contact patch, $v$ is creepage, $A$ is the contact area, and $W$ is the wear rate. Creepage is dimensionless, and is calculated as the relative velocity of the contact point of the wheel respect to the rail (considering rigid bodies) divided by the nominal velocity of the vehicle. For computation of creep forces, it is necessary to use a contact theory to establish the relationship between these tangential forces and the creepages emerging in contact patch. In this paper the FASTSIM subroutine was used [10], which obtains more accurate results than other theories.

There are more complex wear models which, on the basis of discretisation of the contact patch, differentiate zones on the patch that adhere to those that are sliding. It is assumed that wear is only produced in the zone that is sliding, and it is proportional to the normal load and the sliding distance [11-13]. These models are geared towards predicting the evolution of rolling profiles due to wear, since it is this evolution that conditions the vehicle's dynamic behaviour and the very intensity of wear with worn profiles. The proportionality coefficients have been adjusted in these cases on the basis of experimental measurements of wear emerging in real scenarios for both wheels and rails.

(a)

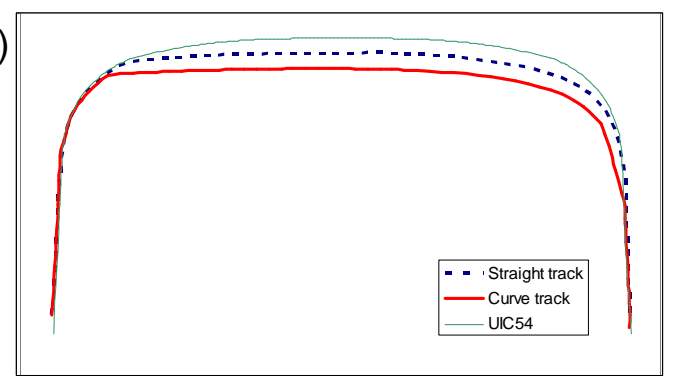

(b)

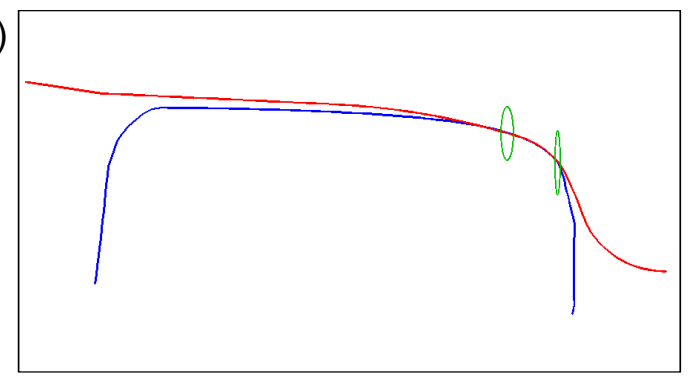

Fig. 3. (a) Theoretical rail profile and as measured experimentally; (b) double contact point with worn profile on the circular section and theoretical wheel profile.

The wear index results presented in this paper were obtained by direct computation of the energy dissipated over the contact patch, and dividing it by the contact area. As it has been seen above, this index is related to the wear rate and it is sufficient to demonstrate the importance of carrying out 3-D analysis of contact when the vehicle is negotiating a tight curve and a double contact point emerges, that is the aim of the paper. Analyses show 
that in these situations accurate location of 3-D contact patches leads to higher wear indices than those calculated using simpler or single-point contact models.

\section{Results obtained for a bogie negotiating a tight curve}

The 3-D multi-point elastic contact model was used to calculate the wear caused by a railway bogie moving over a real curve of radius $80 \mathrm{~m}$. The section analysed was a suburban metric-gauge track outside a station in Bilbao. It is initially straight, with incoming and outgoing transition curves of length $11.25 \mathrm{~m}$, and a circular section with length $64.52 \mathrm{~m}$ and excess gauge width $20 \mathrm{~mm}$. Vehicle speed over the curve was $8.33 \mathrm{~m} / \mathrm{s}$. The theoretic rail profile is UIC-54, and wheel profile DT-14.

The rail profile analysed is not the theoretical profile. Experimental measurements were carried out using a Miniprof Rail device, and a number of definer points were obtained that were subsequently used for interpolation based on spline curves. Figure 3 shows the profiles measured on the straight section and on the circular section. In both cases it may be observed how the rail is worn, but that wear is greater on the curving section, particularly on the inner corner, where there is contact with the wheel flange, producing more intense wear.

3-D contact tables with worn rail profiles and a DT-14 wheel profile were constructed to carry out a dynamic simulation of the vehicle on the curve and ascertain the amount of wear that will be produced, and where this will be most intense. Figure 4 shows the result obtained for the lateral displacement and angle of attack over time for both bogie wheelsets. The 3-D contact model was used, and it was ascertained that the first wheelset makes contact with the flange on the rail head, creating a double contact point scenario as shown in Figure $1 \mathrm{~b}$.

(a)

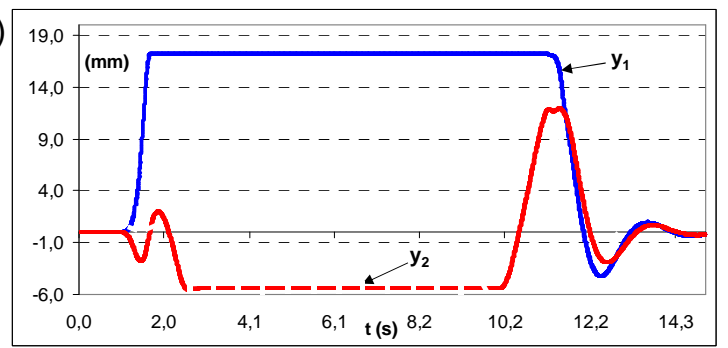

(b)

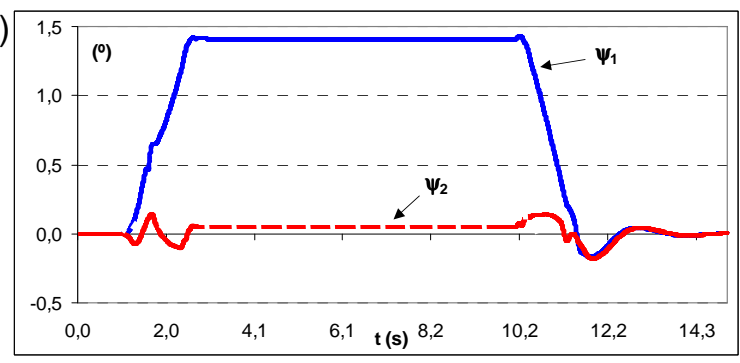

Fig. 4. (a) Lateral displacement over time of the two bogie wheelsets moving over a curve of radius $80 \mathrm{~m}$; (b) angle of attack.

On this curve the front wheelset's angle of attack becomes quite large, almost $1.5^{\circ}$, and this means the second contact point over the flange is not located on the wheel's vertical diametral section. Thus the creep forces, the forces associated with them, and wear indices will be greater than those which would emerge in a contact patch localised with a 2-D analysis of the wheel. To determine and quantify this difference, Figure 5 shows the 
wear index over time calculated in the contact patches on the outer wheel of the first wheelset. The results obtained with the 3-D contact model presented in this paper are compared with those obtained if no account is taken of the angle of attack using a 2-D model.

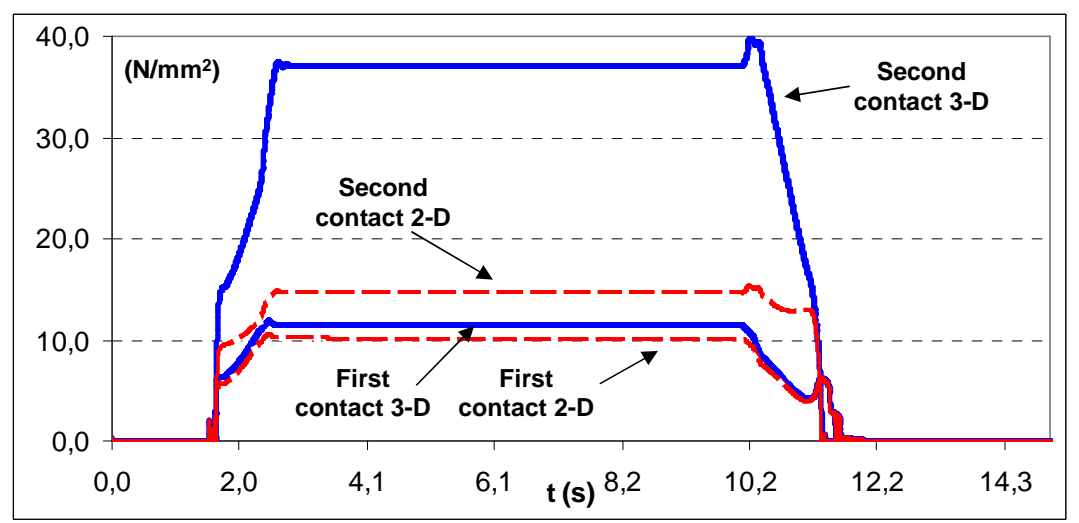

Fig. 5. Wear indices calculated using 2-D (dashed lines) and 3-D (solid lines) contact models over time, for contact on the wheel thread (first contact) and contact on the flange (second contact).

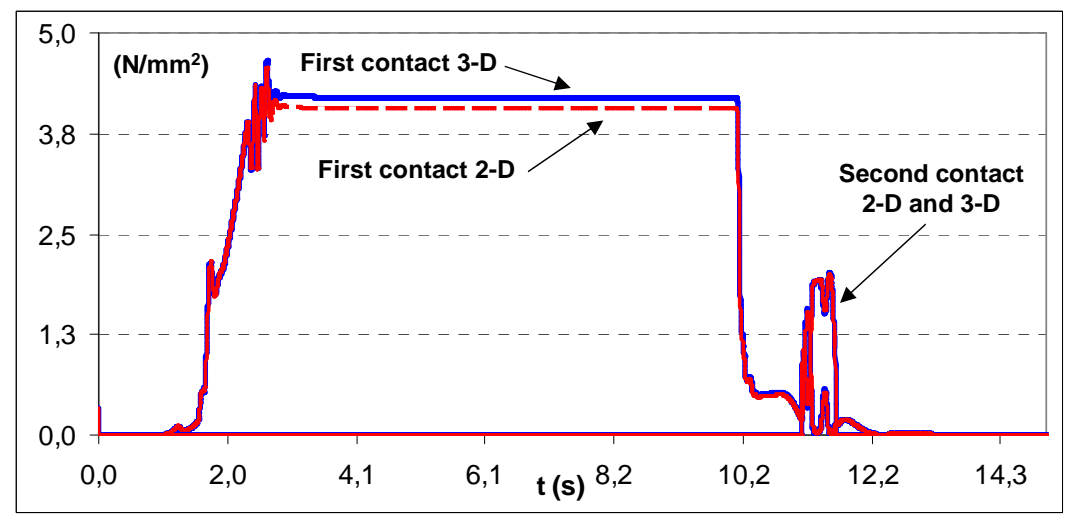

Fig. 6. Wear indices calculated using 2-D (dashed lines) and 3-D (solid lines) contact models over time on areas of contact with the outside wheel on the rear wheelset.

As can be observed, the difference is quite small in the contact patch on the wheel thread. This is because the wheel's contact angle in this area is small, and the contact patch does not have much separation from the wheel's vertical diametral section when the yaw angle increases. Whenever the wheelset reaches a certain level of lateral displacement, however, the gap between the wheel flange and the rail head disappears and a second simultaneous contact point emerges over the wheel flange. Here the contact angle is large $\left(70^{\circ}\right.$ for the DT-14 wheel), and if the wheelset is rotating at a high angle of attack, the contact patch is forward. Figure 5 shows that the wear index 


\section{Javier Santamaria / Wear}

calculated in this contact area with a 3-D model is much higher than that obtained using a 2-D model. Moreover, the wear caused in this second contact area over the flange is much higher than in the first one, as Figure 5 shows, and so this example illustrates the importance of using 3-D analysis of contact on tight curves.

Figure 6 also shows wear indices, calculated for the outer wheel on the second wheelset. Wear is much lower than on the first wheelset on the bogie, mainly because contact occurs on areas of the wheel at a greater distance from the flange. There is, in fact, a brief moment of contact with the flange on the outgoing transition curve, shown as the second contact, and this is associated with a small normal force, and therefore with a low wear index. In both cases it may be observed that the differences between the results obtained using 2-D and 3-D models are very small. This is because the angle of attack is not as large as on the first wheelset, and the contact patches are in proximity to the wheel's vertical diametral section.

Figure 7 shows, over the rail profile, the level of wear calculated with the 3-D contact model along the entire section analysed (transition curves and circular section). It must be borne in mind that the simulations performed take no account of irregularities on the track, which would produce a wider distribution of wear indices over the entire rail profile. It is clear that the higher wear indices are mostly grouped around the inner corner of the rail, the area in contact with the wheel flange on the first wheelset on the circular section. It is at this contact point and in this scenario that the most significant value is found for wear. The profile of the worn rail as measured experimentally shows that it is in fact this area which produces most wear.

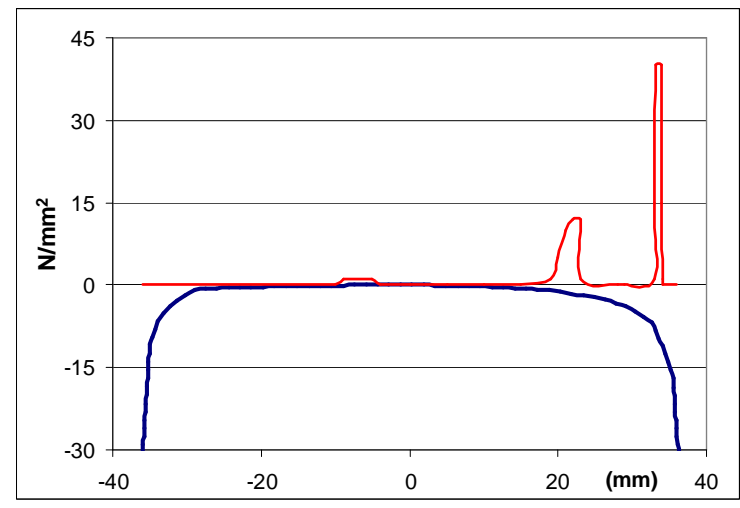

Fig. 7. Wear indices obtained on the profile of the worn rail.

\section{Conclusions}

This paper demonstrates the importance of solving the problem of 3-D wheel-rail contact for an estimation of the levels of wear that will emerge when the vehicle is negotiating tight curves. In these cases the front wheelset's angle of attack tends to adopt high values that have a considerable effect on the localisation of contact patches, 


\section{Javier Santamaria / Wear}

increasing creep and wear indices. A 3-D contact model must be used for proper location of the contact patches, and thus obtain more accurate wear indicators. The paper proposes a 3-D elastic contact model between the wheel and the rail causing two or more simultaneous contacts on the same wheel, based on look-up tables for greater computational efficiency. A real example was worked through to obtain wear levels for both 2-D and 3-D analysis, ascertaining that the results are in fact extremely different and that the contact model is particularly useful for this kind of scenario. These differences disappear when the wheelset's angle of attack is not so high (wider curves or rear wheelsets).

\section{Acknowledgements}

The authors wish to thank the Spanish Ministry of Development for the finance received through the $T$ 83/2006 research project. The authors also acknowledge the financial help received from the Department of Education, Universities and Research of the Basque Government.

\section{References}

[1] S. Iwnicki, Handbook of railway vehicle dynamics, Taylor \& Francis Group, U.K., 2006.

[2] A.A. Shabana, K.E. Zaazaa, J.L. Escalona, J.R. Sany, Modeling Two-Point Wheel/Rail Contacts Using Constraint and Elastic-Force Approaches, J. Sound and Vibration. 269 (2004) 295-325.

[3] V.K. Garj, R.V. Dukkipati, Dynamics of Railway Vehicle Systems, Academic Press, Canada, 1984

[4] K.L. Johnson, , Contact Mechanics ,Cambridge: Cambridge University Press, 1985

[5] J. Santamaría, E.G. Vadillo, J. Gómez, A comprehensive method for the elastic calculation of the two-point wheel-rail contact, Vehicle System Dynamics. 44 (2006) 240-250.

[6] T.G. Pearce, N.D. Sherrat, Prediction of wheel profile wear, Wear. 144 (1991) 343-351.

[7] BBR, Introduction to Railway Vehicle Dynamics, Traction and Rolling Stock Group of British Rail Research, London, 1996.

[8] F. Braghin, R. Lewis, R.S. Dwyer-Joyce, S. Bruni, A mathematical model to predict railway wheel profile evolution due to wear, Wear. 261 (2006) 1253-1264

[9] R. Lewis, U. Olofsson, Mapping rail wear regimes and transitions, Wear. 257 (2004) 721-729

[10] J.J. Kalker, A fast algorithm for the simplified theory of rolling contact, Vehicle System Dynamics,.11 (1982) 1-13.

[11] J.F. Archard, Contact and rubbing of flat surfaces, J. Appl. Phys. 24 (1953) 981-988.

[12] R. Enblom, M. Berg, Impact of non-elliptic contact modelling in wheel wear simulation, Wear. 265 (2008) $1532-1541$.

[13] T. Telliskivi, Simulation of wear in a rolling-sliding contact by a semi-Winkler model and the Archard's wear law, Wear. 256 (2004) 817 831 . 